\title{
Bandwidth Estimation for Admission Control in MANET: Review and Conceptual MANET Admission Control Framework
}

\author{
Folayo Aina $^{1}$, Sufian Yousef ${ }^{1}$, and Opeyemi Osanaiye ${ }^{2}$ \\ ${ }^{1}$ Anglia Ruskin University, Chelmsford, CM1 1SQ, UK \\ ${ }^{2}$ Federal University of Technology, Minna. P.M.B, 65 Niger State, Nigeria \\ folayo.aina@pgr.anglia.ac.uk
}

\begin{abstract}
Mobile ad-hoc network (MANET) is one of the main technologies for the next generation wireless networking because of the positive impact it poses over other wireless networks having undergone rapid progress, which has inspired many applications. However, providing quality of service (QoS) assurance to MANET is hard because of the unpredictable nature of the wireless medium, contention problem amongst the channel, mobility problem and lack of central co-ordinator. Admission control is therefore seen as one of the methods for providing QoS. Admission control aim at estimating the network resource states and decides whether to admit a session without assuring more resources bandwidth space than what is available, to avoid the violation of any rules that has been previously made. Some recent solution considered the MAC layer back-off impact due to collision as well as the non-synchronization between the sender and receiver when estimating the available bandwidth. None of the previous work proposed a technique that sends a HELLO packet to its one-hop neighbours which further aggregates to the rest of the nodes to retrieve the available bandwidth on a carrier sensing region, in order to limit the impact of additional overhead of the carrier sensing multiple access with collision avoidance (CSMA/CA). Also, none of the existing solution has properly addressed the channel idle time dependency between the sending node and the receiving node by differentiating the $B U S Y$ state from the SENSE BUSY states and the IDLE state caused by an empty queue. This paper, therefore reviews the bandwidth estimation techniques for admission control for MANET. The bandwidth estimation techniques for admission control have been categorized into two, active and passive estimation. An outline of each technique has been discussed as well as the proposed conceptual framework. The contribution as identified in this research work is the proposal of conceptual framework that adapts the following into the bandwidth estimation for admission control in MANET: (i) HELLO packet advertisement to one hop which further aggregates to retrieve the available bandwidth on the carrier sensing region, (ii) Considering the channel idle time measurement by differentiating the channel busy state from channel sensing state and regarding an empty queue as an idle state. Future research directions are also outlined.
\end{abstract}

Keywords: Admission Control, Bandwidth, QoS, MANET.

\section{Introduction}

Over the past 15 years, the attention given to mobile ad-hoc network (MANET) has significantly increased. MANET have been developed to provide flexible and spontaneous communication in locations where there are limited or lack of centralized infrastructure. Internet access can be provided by a gateway node, but MANET users collaborates by sharing contents and messages amongst themselves. Application areas of MANETs are battlefields, temporary gathering i.e. conferences, virtual classrooms and construction network site [1]. Developing country, in which people live in areas where there is less infrastructure can extremely gain from MANET technology. As a matter of fact, the project of "One Laptop Per Child" is bringing about the highest realworld MANET like networks till date [1]. Recently designed laptops and personal digital assistants (PDA) has 802.11- compliance air interface embedded in it with the intention of operating them in an ad-hoc mode, therefore, 802.11 wireless is a major enabling technology of MANET. QoS provision to MANET users possess concern to the service provider as well as the service user. Tasks, especially real-time applications, require QoS to enhance its communication (i.e. multimedia data). Nodes must therefore cooperate with one another to guarantee effective QoS. The cooperation must include the endpoint flow policing as well as admission control implementation along the route, to prevent network violation of initially made policy. The aim of deployed QoS support is to provide guaranteed application support in term of delay, jitter, throughput, bandwidth, etc. To ensure this, the MAC layer takes the responsibility of allocating resources at different nodes, while the network layer considers resources along the entire communication route. The wireless network support for QoS when compared with its wired counterpart is not trivial, due to its lack of infrastructure and sharing of resources and medium [3] [4]. A 
mechanism that provides QoS assurance is known as admission control. Admission controls aim is to decide on what kind of data application that can be admitted into the network without having to promise more resources of unavailable spaces in order not to violate any previously made guarantees [5]. Admission control can control the allocations and usage of network resources for several applications that requires additional services. Admission Control is therefore seen as a component that needs to allow resources such as bandwidth to be used only when it is available [6].

Some recent solution considered the MAC layer back-off impact due to collision as well as the non-synchronization of the sending and receiving node during the available bandwidth estimation process. None of the previous work proposed a technique that sends a HELLO packet to its one-hop neighbours which further aggregates to the rest of the nodes to retrieve the available bandwidth on a carrier sensing region, in order to limit the impact of additional overhead of the carrier sensing multiple access with collision avoidance (CSMA/CA). Also, none of the existing solution has properly addressed the channel idle time dependency between the sending and the receiving node by differentiating the BUSY state from the SENSE BUSY states and the IDLE state caused by an empty queue

This paper reviews the common bandwidth estimation technique for admission control in MANET and it categorizes such estimation into active and passive bandwidth estimation technique. Furthermore, this paper discusses the active and the passive protocol sub-division highlights the protocol pertaining to each of the techniques that were found in the literature. Thereafter, a table summarizing the bandwidth estimation technique for admission control in MANET with their innovations were clearly outlined.

This paper reviews publications that exist in literature up until 2018 from ACM digital Library, IEEE Xplore, Google Scholar, ScienceDirect, Springer, and the, using keywords such as "Admission control in MANET, "Bandwidth estimation for admission control" "QoS in MANET" and "Admission control survey". Some survey works have been conducted in regard to this topic, however, to the best of our knowledge, no extensive survey exist as regards bandwidth estimation technique for admission control in MANET.

The contribution as identified in this research work is the proposal of conceptual framework that adapts the following into the bandwidth estimation for admission control in MANET: (i) HELLO packet advertisement to one hop which further aggregates to retrieve the available bandwidth on the carrier sensing region, (ii) Considering the channel idle time measurement by differentiating the channel busy state from channel sensing state and regarding an empty queue as an idle state.

This paper is arranged as follows: Section 2 discusses the bandwidth estimation for admission control. Section 2.1 describes the active bandwidth estimation techniques and their sub-divisions while protocols under the active bandwidth estimation technique was also outlined in this chapter. Section 2.2 describes the passive bandwidth estimation techniques as well as its sub-divisions together with its protocol. Section 3 presents a general discussion while in section 4 the paper was concluded.

\section{Bandwidth Estimation for Admission Control}

There have been several approaches proposed in the literature for available bandwidth estimation with admission control implementation. Author [7] [8] classified the bandwidth estimation techniques into passive (i.e. nonintrusive) estimation and active (i.e. intrusive) probing. In [9] [10], bandwidth estimation techniques were categorized into active probing, mathematical model based, and calculation based passive estimation. The author in [11] had a different approach by classifying it into self-congestion and model-based approach, while [12] classified the bandwidth estimation technique into algorithm intended for a precise network usually with QoS guarantee, algorithm that uses probe packet with pre-determined spacing, and algorithms focusing on video streaming where a client-server assumption is made. Researchers have differently classified bandwidth estimation techniques; however, they perform the same role notwithstanding their different nomenclatures. We therefore argue that classification of bandwidth estimation into active technique and passive technique as categorized by [7] and [8] will simplify the readers understanding of the bandwidth estimation process for admission control.

\subsection{Active Bandwidth Estimation Technique}

In active bandwidth estimation, a dummy packet known as probe packet is transmitted along the network path at different data transmission rates. The available bandwidth estimation along a path is therefore carried out by estimating the different time of arrival [13]. The above technique adds probing traffic and can possibly degrade 
the existing flow performance [14]. The main objective of the active technique is to observe the network characteristics by introducing the probe packet.

Most of the previous work classified the active available bandwidth estimation for admission control into single packet/one packet and packet pair [3], while other classifications of active technique are different but with the same role. In [15] the active available bandwidth estimation technique is classified into isolated probing, direct probing, and iterative probing. The author in [16] classified it into direct probing and iterative probing technique. In [17], the active available bandwidth estimation was classified into direct probing, iterative probing, and mixed techniques, while [18] classified it into packet dispersion measurement (PDM), probe gap model (PGM) and probe rate model (PRM). In [19] the active technique was classified into variable packet size (VPS) probing, packet pair/train dispersion model (PPTD), self-loading periodic streams (SLoPS) and train of packet pairs (TOPP).

It was observed in [15] that isolated probing and probe delay model are the same as single-packet probing technique. In [20] the author regards PRM and iterative to be a self-loading technique. Also, [20] regards PGM and direct probing to be a packet-pair dispersion technique.

We therefore argue that classification of active bandwidth estimation into single packet/one packet and packet pair as categorized by [3] will simplify the readers understanding of the active bandwidth estimation process. A diagram showing the subdivision of active protocol is shown figure 1below:

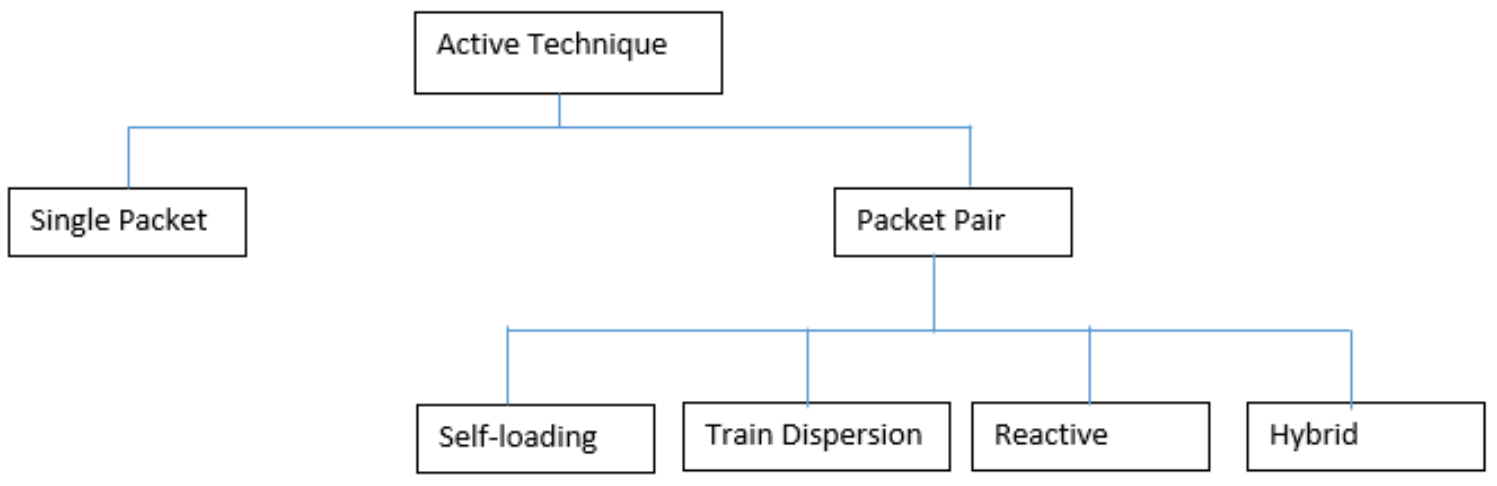

Figure1: Active bandwidth estimation

Single Packet Active Technique: In this active technique, one probe packet at a predefined time interval is injected into the network in to measure the delay. Link capacity is measured rather than end-to-end path capacity by making use of the interval time difference between the round-trip time in the probe packet from one end of the link to the other [21]. The packet transmission time $\mathrm{t}=(\mathrm{P} / \mathrm{b})+\mathrm{l}$, where $\mathrm{P}=$ packet size, $\mathrm{b}=$ link bandwidth, and 1 is the fixed latency. If the round-trip time and the probe packet size is known the bandwidth can be estimated for a giving fixed latency of a link. Tools that exist under single packet active probing are as follows; clink [22], pathchar [23], tailgating [24], and pchar [25]. The only protocol implemented for the estimation of bandwidth using single probe packet is variable packet size probing [26].

In [26], Variable packet size probing was proposed. The capacity measurement along the end path is carried out by this protocol, and it makes use of the round-trip time from the sending node through to each hop along the path with respect to the probe packet sizes. In variable packet size probing, a probe packet frame is transmitted from a source node to the network layer along the path of each hop in a continuous way at a predefined interval. The time-to-live (TTL) field of the IP header in the probe packet forcefully terminate the probe packet when it gets to certain target hop. Internet control message (ICMP) time exceeded error message is sent back to the sending node from each of the hop lies around that path.

Packet Pair Active Technique: In this active technique, two probe packets known as packet pair are transmitted back-to back towards the target link, and this generates an echo back to the sending node. The space as seen in figure 2 between packet P1 and P2 is always based on the bottleneck link which is kept by a bandwidth link with 
higher value [27]. A packet within the packet pair that arrives at the target node have a specific time space between each packet which is specified by $\Delta i n$. Having interacted with the traffics from cross layer coming from various sources, packets exit the output queue with changed time separation which is stored as $\Delta$ out. The packet-pair active probing technique can therefore be further classified into: self-loading packet-pair active probing, packet pair/train dispersion active probing, reactive packet-pair active probing, and hybrid packet-pair active probing.

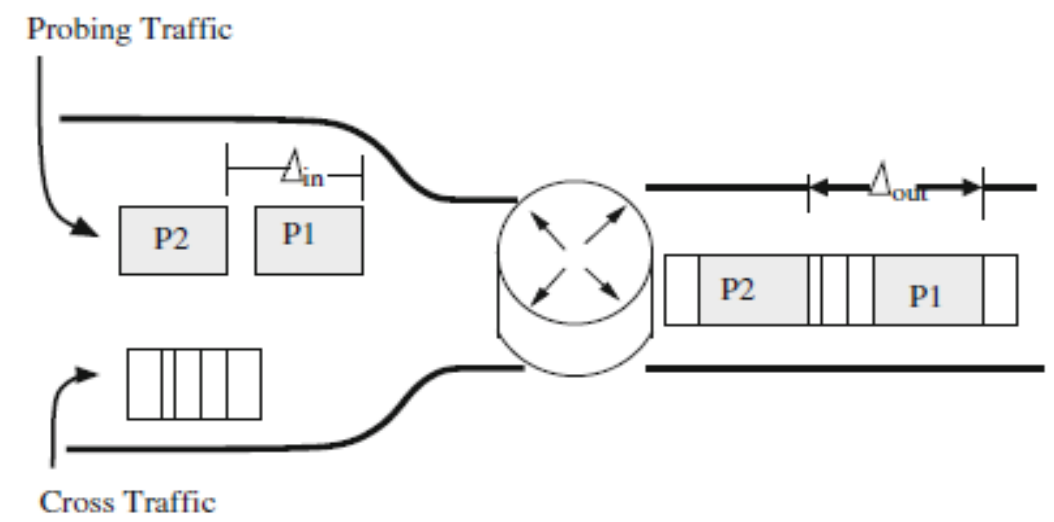

Effect of probing packets at node with time-separation

Figure 2: Effect of packet probing at nodes having time separation

Self-loading packet-pair active probing: In this packet pair active probing technique, trains of probe packets are iteratively transmitted into the network channel at different data rate. When compared with a non-iterative packet pair active probing, more probing bits are required which yields a more accurate estimation. The probing bit requirement results in severe intrusiveness and long measurement time. If the rate of sending the probe packet is faster than the available bandwidth, the probe packet will queue, this therefore lead to an increase in the end-toend delay [28]. The available bandwidth estimation is therefore measured using the variation of delay encountered at the receiver's node. Protocols that falls within the self-loading packet-pair in literature is as follows: MinProbe [14], self-loading decreasing rate train (SLDRT) [30], probabilistic available bandwidth (PAB) [31], Self-loading periodic stream (SLoP) [16], Train of packet pair (TOPP) [19], Packet train pair (PTP) [28] [29], etc.

MinProbe was proposed by [14]. This protocol is an active protocol that measures the available bandwidth of a node with high fidelity, minimal cost and in user space.

In [30], Self-loading decreasing rate train (SLDRT) was proposed. The available bandwidth estimation measurement is carried out by making use of a single diminishing rate packet train under a cross-traffic. This was achieved by sending a single decreasing packet train rate with probe packet. These packets therefore stores at the tight link causing the one-way delay of successive packets at the receiver which shows an increase in trend. With the packet-train rate decreasing, the tight link congestion is gradually eliminated, and the one-way delay will show a decrease in trend. Eventually, the one-way delay remains approximately stable when the probe packet trains input rate is the same as the available bandwidth. SLDRT protocol deduces the available bandwidth by making use of the whole probe packet trains rather than considering the rate of individual packet. By doing this, the bias measurement due to busty traffic can be effectively eradicated.

The authors of [31] proposed probabilistic available bandwidth (PAB). This protocol estimates the probabilistic available bandwidth of multiple path in a network. This was achieved by determining the highest input rate for which a traffic flow can send to achieve an output rate that is nearly equivalent to the input rate with specific probability. In PAB three different tasks are executed: firstly, it probes a path and present a measured outcome. Secondly computes the marginal posterior of the probabilistic available bandwidth path from measurement outcome by running propagation on the factor graph and establish confidence interval for the probabilistic available bandwidth, Finally, it identifies measurement (by choosing the path) at each iteration that minimizes the network overhead.

Packet Pair/Train Dispersion active probing: This protocol can also be referred to as direct probing or probe gap model. It is a fast and lightweight available bandwidth measurement. In packet pair/train dispersion active probing, 
the cross traffic goes in the middle of the probe packet and transmits them as shown figure 2. The available bandwidth estimation is done by mathematically computing the measured sent and received gaps between probe packets. The list of the protocols that falls under packet pair/train dispersion active protocol in literature are: twoway available bandwidth estimation(TWABE) [32], gaps of non-adjacent probe packet (GNAPP) [8], network link characteristics using packet pair dispersion (NLCPPD) [33], new enhanced available bandwidth measurement technique (NEXT) [34], new enhanced available bandwidth measurement technique extension (NEXT-V2) [35], new enhanced available bandwidth measurement technique extension with piggybacking (NEXTV2 with piggybacking), WBest [36], RT-WABEST [37], initial gap increase (IGI) [38] [39], adaptive available bandwidth estimation (AABE) [40], etc.

The authors in [32] proposed a two-way available bandwidth estimation (TWABE). This protocol estimates the available bandwidth of the up-link as well as the down-link by using the ICMP and traceroute timestamp concept. In this protocol, the ICMP implements the traceroute which is used in calculating the path length from the sender to the receiver. When it gets to the ith round $(I=1,2, \ldots \ldots, \mathrm{H})$, an NICMP probe packet with $\mathrm{TTL}=\mathrm{i}$ is forwarded by the sender to the receiver.

In [8], gaps of non-adjacent probe packet (GNAPP) was proposed. This protocol bidirectionally measures the available bandwidth in a similar way with TWABE. It evaluates the available bandwidth of both the uplink and the down-link tight link path. The application area of most probing techniques does not focus on multimedia network streaming they only perform the evaluation of a paths available bandwidth along a specific direction, like from the source to the destination. GNAPP makes use of traceroute and modified ping program which uses an ICMP timestamp.

Authors in [33] proposed network link characteristics using packet pair dispersion (NLCPPD). This protocol analyses dispersion of packet based probing technique within a unicast and multicast tree and develops a theoretical model of discreate time queue by considering the characteristics of the link. The method used in NLCPPD allows packet pair probes with a given space in between them to be inserted at the source node. The probe packet allows a discrete time queue on one path or on the multicast tree. For a single queue with a specific space in between the input probes, the conditional distribution separation between the queue output probes in terms of the arrival process distribution is derived.

New enhanced available bandwidth measurement technique (NEXT) was proposed by [34]. NEXT is a probing technique with a rate adjustment algorithm used in estimating end-to-end available bandwidth on a network part. Its concept is based on self-inducing congestion and a probe train structure that allows a packet to be frequently sampled on a given region than another region. The highest sample region allows the algorithm to find a more accurate turning point. Whenever the dynamic available bandwidth is outside the highest sampled region, the lower and upper packet stream rate is readjusted to fit the dynamic available bandwidth into the region. The spread factor is used in adjusting the range between the lower and the upper rate to keep the packet number less, and the available bandwidth is measured intrusively.

The authors of [35] proposed a new enhanced available bandwidth measurement technique extension (NEXT-V2) which is an active protocol. This protocol was said to be an extended version of NEXT, that effectively measures the end-to-end available bandwidth within a fixed wireless channel. The structure of this protocol is more like a packet train with an optimal rate adjustment and a modification of excursion detection algorithm which is used to distinguish the available bandwidth with higher accuracy, less overhead and less convergence time.

New enhanced available bandwidth measurement technique extension with piggybacking (NEXTV2 with piggybacking) was proposed by [34]. In this protocol, estimation is based on a proxy technique that sends data application by piggybacking inside the probing packets resulting in less overhead.

Authors in [36] proposed a wireless bandwidth estimation tool (WBest). This protocol has a two-stage algorithm, namely; packet pair technique that measures the capacity of a link where the last hop is the wireless LAN (WLAN) and a packet train technique that measures the throughput achieved in order to know the available bandwidth. The parameters of WBest are optimised with trade-off of accuracy, convergence time and intrusiveness. WBest avoids using a search algorithm for the detection of the available bandwidth, therefore it statistically detects the available link fraction to limit the delay impact of wireless channel random error.

Round-trip wireless available bandwidth estimation tool (RT-WABest) was proposed in [37]. It was designed based on round trip time measurement with two stage algorithms. The first stage uses packet pair dispersion 
method for the estimating path capacity while the second stage transmits a packet train within the network to infer available bandwidth.

Reactive packet-pair active probing (RPPAP): This is another available bandwidth estimation technique. In this technique, for a reactive bandwidth measurement to be activated, a probe packet is transmitted by a sending node to the receiving node. If the sender does not get the acknowledged probe packet before the time out period, the source node resends the probe packet. Once the receiving node receives the probe packets that has been sent by the sender node, it acknowledges it by periodically transmitting lots of back to back probe packet on all the available paths to the source node. The packet thereafter travels along the path from the sending node to the receiving node and produces spaces in between them. The following are the protocols that are identified to exist under RPPAP which will be discussed in this study: Multi-Rate available BE in Real-Time (MR-BART) [41], minimal backlogging techniques (MiBT) [42], distributed admission control for MANET environments (DACME) [43], reactive bandwidth measurement in 802.11 networks (RBM) [44], bandwidth available in realtime (BART) [45] [46], etc.

The authors in [42] proposed minimal backlogging technique (MiBT). The available bandwidth estimation is carried out by making use of statistics of the probing traffic service rate. MiBT avoids using probe gap model and probe rate model. The probing traffic service rate statistics is a constant available bandwidth estimation process for a $\mathrm{G} / \mathrm{G} / 1$ queuing system under a minimal backlogging condition which supports MiBT on a theoretical basis. For MiBT to be emulated in an actual multi-hop network, the minimal backlogging condition or probing rate closer to the available bandwidth based on the length of the busy period is detected. In order to ensure a minimal backlogging condition is maintained, the probe rate adaptively changes.

In [47] PATHCOS++ was proposed. With PATHCOS++ the available bandwidth of a channels end-to-end path is estimated by sending a train of time stamp probe packet from a sending node to a receiving node and integrating the advantages of probe rate model and probe gap model-based techniques. It consists of congestion mechanism gaps that is induced for available bandwidth estimation. Changes in one-way delay of the probe packet is observed by the receiver and analysis are conducted based on the mechanism.

For any wireless ad-hoc network the active measurement technique is not ideal based on the following [48]:

- In an active estimation technique, probe packet is used for measurement of the available bandwidth between the source and the destination node. If the source and destination node number is high, there will be many probe packets sent within the network end to end pair, therefore requiring a higher amount of bandwidth [48].

- Because of the changing nature of wireless channel, the network topology is unstable when compared to wired link topology. Therefore, the active bandwidth estimation will have to perform its estimation at a higher frequency, resulting in additional usage of bandwidth [48].

- The active bandwidth estimation introduces extra overhead, affect the accuracy, and degrades the network performance of the bandwidth estimation. Therefore, the active bandwidth estimation approach is not the best choice for measurement in wireless networks [49].

\subsection{Passive Bandwidth Estimation Technique}

In [50], passive estimation is referred to as a calculation-based technique. Passive bandwidth estimation technique does not inject any probe packet into the network when estimating the required available bandwidth. Dispersion and delay are monitored for the acknowledgement and flow of data without using any probe packet. This form of estimation works with earlier generated information traces collected. The local information on bandwidth utilized is used for calculating the available bandwidth and it is exchanged using the local broadcast. Passive bandwidth estimation can be divided into two, namely; generic passive technique (GPT) and proactive passive technique (PPT). A diagram showing the subdivision of passive protocol is shown in figure 3 below: 


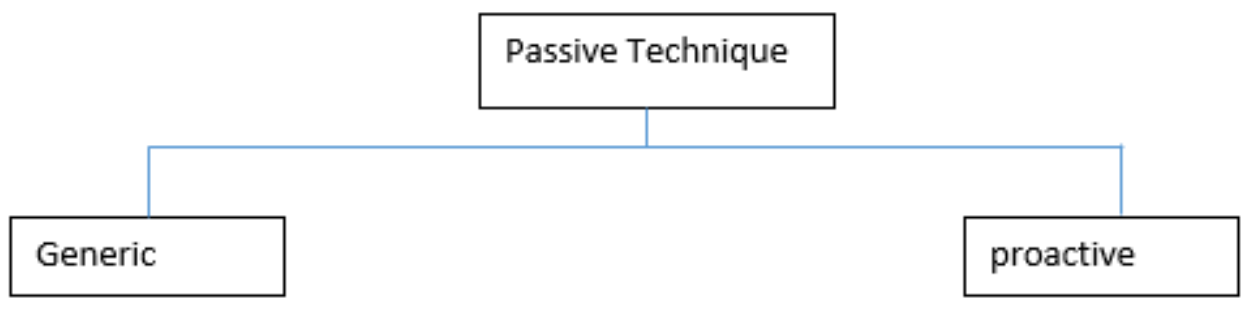

Figure3: Passive Technique

Generic passive technique (GPT): This protocol requires a probability distribution function (PDF) of a TCP flow packets interarrival time [7]. The PDF shows the behaviour of spike, spike bump, spike train and train of spike bumps. The characteristics of GPT is described as a bottleneck having no cross-traffic. The types of GPT protocol are; estimation of available bandwidth ratio of a remote link or path segments (EABRRL) [51], TCP Vegas (TCPV) [52], and TCP Westwood (TCPW) [53].

In [51], Estimation of available bandwidth ratio of a remote link or path segments (EABRRL) was proposed. This protocol performs an estimate of the available bandwidth ratio along the remote path or link even though it is not deployed at the remote node. EABRRL measures the delay of a segment path remotely by monitoring the node. Here, two ICMP timestamp streams of packets are sent to the end-to-end node of a target link in accordance with Poisson process. The one-way delay is measured by computing the difference between the packet sending time and the value of timestamp received from remote nodes. It also extracts the queuing delay component from the delay measured and estimates the available bandwidth product ratio of a given path on a link. The available bandwidth is thereafter inferred from the ratio of the available bandwidth products.

Proactive passive technique (PPT): Proactive passive technic is non-intrusive because there is no frequent exchange of HELLO packet. This protocol only considers the MAC overhead during the available bandwidth estimation. The available bandwidth helps in network selection in an heterogenous network. Parts of the protocols that comes under PPT are: available BE (ABE) [54], improved available bandwidth (IAB) [50], cognitive passive estimation of available bandwidth (cPEAB) [10], accurate passive bandwidth estimation (APBE) [9] distributed available BE (DABE) [55] [56], QoS enabled routing in MANETs (QoS- AODV) [57], contention-aware admission control protocol CACP [58], adaptive admission control (AAC) [59].

The authors in [54] proposed an available bandwidth-based flow admission control (ABE) algorithm for wireless network. Estimation of the available bandwidth is done by using the wireless channel sensing mechanism, where consideration is given to both virtual and physical carrier sensing, and the various wireless CSMA/CA MAC layer interframe spacing. They argued that when the channel activities are measured by taking into consideration the amount of time spent in the physical and virtual carrier sensing with different interframe space, this will result in over-estimating the available bandwidth. This occurs as a result of the non-synchronization between the source node and the destination node within an ad-hoc network.

Authors in [50] proposed an improved available bandwidth (IAB). The improved available bandwidth estimation measures the available bandwidth of a giving link for QoS support in wireless ad-hoc network. It considers synchronization between the source and the destination node by distinguishing the busy rate caused by the transmitting and receiving nodes from those caused by the sensing nodes. It also improved the accuracy of estimating the overlapping probability of the idle channel time of two adjacent nodes. For a node to be termed BUSY, it must be in either a receiving state or transmission state. A node is termed SENSE BUSY when it is in a sensing state. If the node is not in any of these states, it means the node is IDLE. The drawback of this technique is similar to those mentioned in [50].

Cognitive passive estimation of available bandwidth (cPEAB) was proposed by [10]. This protocol estimates the available bandwidth of a network in an overlapped WiFi WLANs environment. It considered the additional overhead due to by acknowledgement packet, which was ignored in AAC and ABE. Furthermore, it performs the estimation of available bandwidth by measuring the value of waiting and back off delay, packet collision 
probability, acknowledgment delay, and channel idle time. $\mathrm{CPEAB}$ also considers the hidden and exposed node to have a more accurate available bandwidth measurement. The disadvantage of this measuring technique is that the intra-flow contention count calculation may not provide a right contention count all the time. Also, the dependency of the channel idle time ratio only differentiates between the busy and sense busy and did not consider an empty queue to be an idle channel time period. Additional retransmission and contention window overheads were also not considered. Lastly, to retrieve the available bandwidth on a carrier sensing, HELLO packet is broadcasted to two hop neighbour which tends to flood the network to increase the network overhead.

Author in [9] proposed an accurate passive bandwidth estimation (APBE). In APBE, the available bandwidth is estimated by considering request to send (RTS) and clear to send (CTS) overhead. It measures correctly the value of DIFS and back-off, the packet collision probability, the acknowledgment delay and the channel idle time. It is calculated as:

$\mathrm{R} / \mathrm{C}_{\text {ohd }}=((\mathrm{RTS}+\mathrm{CTS})+(2 \mathrm{X}$ SIFS $)) / \mathrm{T}$.

To estimate the available bandwidth,

$\mathrm{ABW}=(1-\mathrm{K}) \mathrm{X}(1-\mathrm{R} / \mathrm{C}) \mathrm{X}(1-\mathrm{ACK}) \mathrm{X}\left(1-\mathrm{P}_{\mathrm{c}}\right) \mathrm{X}\left(\mathrm{T}_{\mathrm{i}} / \mathrm{T}\right) \mathrm{X} \mathrm{C}$

The author in [44] improved on the work proposed by [54] by adding to its algorithm, a retransmission mechanism and back-off overhead. The weakness of this technique is that the contention window overhead was ignored with higher data traffic load in the network. Also, the mathematical model assumption specified may not concur with the actual network.

Authors in [48] proposed a proactive bandwidth estimation (PABE) for wireless based network. PABE is a measurement-based available bandwidth estimation method and flow control admission control technique. Rather than of making use of models to predict the collision and back-off, empirical gathering data was adopted for predicting any additional back-off overhead. Besides, it used the value of the expected future data traffic load to predict additional overhead instead of using the value of the current data traffic. The drawback of this algorithm is that, if there is an increase in data traffic load within a network, additional retransmission and contention window overheads are not considered. Also, the intra-flow and inter-flow contention count are not calculated appropriately. Lastly, to retrieve the available bandwidth on a carrier sensing, HELLO packet is broadcasted to two hop neighbour which tends to flood the network, therefore increasing the network overhead.

In [50], passive available bandwidth estimation (PABE) was proposed. In this protocol, the effectiveness of the link capacity is considered by analysing the random factor in transmission such as back-off time and the frames retransmission. For the channel idle time ratio to be estimated, a new and low threshold is introduced.

Authors in [3] proposed distributed LaGrange interpolation based available bandwidth estimation (DLI-ABE). In this protocol, the channel idle time synchronisation uses the actual channel utilization and collision rate. Also, the collision probability model makes use of a separate Lagrange interpolation polynomial at each node depending on the behaviour of node.

Available bandwidth estimation method for wireless ad-hoc network with concurrent transmissions (ABCT) was proposed by [60]. This protocol focused on estimating available bandwidth of a medium using the control-gap based concurrent transmission.

Measured based bandwidth estimation technique and flow admission control (BandEst) was proposed by [61. This protocol proactively considers the whole wireless 802.15 .4 's unslotted CSMA-CA MAC layer overheads and considers the future load. It also considers the estimation of intra-flow contention and estimates contention on non-relaying nodes. Additional MAC layer overhead with respect to the increased data traffic load was considered along-side an algorithm that that has to do with concurrent admission request in FIFO was implemented. The drawback of BandEst is that it has a higher overhead because it broadcast to two-hop. BandEst does not also consider the channel idle time dependency. The effect of hidden/exposed collision node on the accuracy of bandwidth estimation has also been neglected by this protocol.

\subsection{Discussion}


Bandwidth estimation is a vital admission control component to enhance QoS in MANET. In the preceding section, we categorized the bandwidth estimation technique into active technique and passive technique. Different nomenclatures have been used for bandwidth estimation categorization by different researchers, however, all those classifications perform similar functions. The bandwidth related metrics as identified in the literature are link/path capacity and available bandwidth estimation at the node [19]. Most research effort appears to have used the active bandwidth estimation technique for estimation of wired link channel while only few attempts were directed towards using the active measurement technique for estimating wireless network because of its inaccuracy in measurement. Active end-to-end available bandwidth estimation introduces extra overhead, affect the accuracy, and degrades the network performance of the bandwidth estimation. Therefore, the active bandwidth estimation approach is not the best choice for measurement in wireless networks [49]. Passive techniques, on the other hand, uses local information such as loss in packet, delay, and congestion situation to monitor the medium of communication. This is done over certain period of time without causing any impact to the existing traffic flow [9] [13]. Passive techniques therefore prevent extra usage of bandwidth channel which may cause more overhead because it does not send probe packet through the network to get information about the available bandwidth.

Having reviewed the literature, we identified that the state-of-the-art available bandwidth for admission control broadcasts to two hop neighbours in order to retrieve the available bandwidth on a carrier sensing region. This tends to create a higher overhead that can possibly be avoided. Also, the channel idle time dependency sensed by the sender and receiver has not been properly addressed, as most previous work did not consider it. The related works that considered the channel idle time dependency only differentiates the BUSY state from the SENSE BUSY states and the IDLE state caused by an empty queue is yet to be addressed. In this section we present our conceptual MANET bandwidth estimation for admission control framework which address how well the available bandwidth can be retrieved on the carrier sensing region without flooding the network with broadcast messages. To retrieve the available bandwidth on a carrier sensing region, the HELLO message only advertises to the firsthop range which further aggregates to other hops. This technique adopted to retrieve the available bandwidth limits the overhead generated by the network. The channel idle time dependency sensed by the sender and receiver has also been addressed by not only differentiating the BUSY state from the SENSE BUSY states but addressing the IDLE state that can be caused by an empty queue.

Table1. Summary of some bandwidth estimation technique for admission control in MANET

\begin{tabular}{|l|l|l|l|l|l|l|l|l|l|}
\hline Year & Protocol & Active & Passive & $\begin{array}{l}\text { BWE at } \\
\text { Path }\end{array}$ & $\begin{array}{l}\text { BWE at } \\
\text { Link }\end{array}$ & $\begin{array}{l}\text { BWE at } \\
\text { Node }\end{array}$ & Accuracy & Overhead & Innovations \\
\hline
\end{tabular}




\begin{tabular}{|c|c|c|c|c|c|c|c|c|c|}
\hline 2008 & 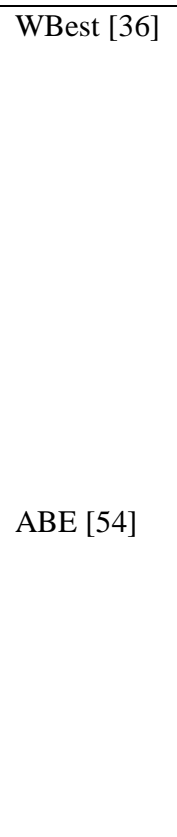 & $\checkmark$ & $\checkmark$ & $\checkmark$ & $\checkmark$ & $\checkmark$ & Medium & 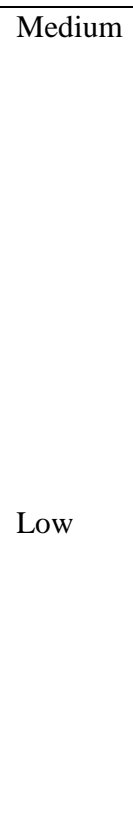 & $\begin{array}{l}\text { It makes use of packet } \\
\text { dispersion model in order not } \\
\text { to depend on search algorithm } \\
\text { for available bandwidth } \\
\text { measurement. It therefore, } \\
\text { statistically estimates the } \\
\text { relative available fraction of } \\
\text { the effective capacity, } \\
\text { mitigation estimation delay } \\
\text { and effect of wireless channel } \\
\text { error. } \\
\text { Considered various retrieval } \\
\text { range as well as making use of } \\
\text { the back-off, waiting time, } \\
\text { channel idle time, } \\
\text { measurement period, } \\
\text { maximum capacity, and } \\
\text { collision probability during } \\
\text { the estimation process. }\end{array}$ \\
\hline 2009 & $\begin{array}{l}\text { TWABE } \\
{[32]} \\
\text { IAB [50] }\end{array}$ & $\checkmark$ & $\checkmark$ & $\checkmark$ & $\checkmark$ & $\checkmark$ & Varies & Medium & $\begin{array}{l}\text { Estimation that considers the } \\
\text { uplink, downlink, and packet } \\
\text { loss environment. } \\
\text { It differentiates the busy state } \\
\text { of a channel caused by } \\
\text { transmission from the sense } \\
\text { busy caused by the carrier } \\
\text { sensing. }\end{array}$ \\
\hline 2010 & $\begin{array}{l}\text { PATHCOS } \\
++[47] \\
\text { cPEAB [10] }\end{array}$ & $\checkmark$ & $\checkmark$ & $\checkmark$ & $\checkmark$ & $\checkmark$ & Varies & Varies & $\begin{array}{l}\text { Provides for self-induced } \\
\text { congestion mechanism as well } \\
\text { as packet gaps. } \\
\text { Additional overhead caused } \\
\text { by acknowledgement frame } \\
\text { was considered which was not } \\
\text { considered in ABE and IAB. } \\
\text { Its innovation is similar to } \\
\text { cPEAB, but it has an addition } \\
\text { of RTS and CTS. }\end{array}$ \\
\hline
\end{tabular}




\begin{tabular}{|c|c|c|c|c|c|c|c|c|}
\hline $2 \mathrm{O} 11$ & $\begin{array}{l}\text { NLCPPD } \\
\text { [33] } \\
\text { PAB [31] } \\
\text { QBEM [13] }\end{array}$ & $\checkmark$ & $\checkmark$ & $\checkmark$ & $\checkmark$ & High & Varies & $\begin{array}{l}\text { It considers the conditional } \\
\text { distribution of separation } \\
\text { between the output probe of a } \\
\text { queue. } \\
\text { Makes use of weighted } \\
\text { entropy/weighted confidence } \\
\text { interval on multipath with } \\
\text { specific rate of probing. } \\
\text { Uses HELLO packet available } \\
\text { bandwidth from the ratio of } \\
\text { busy and free time. }\end{array}$ \\
\hline 2012 & $\begin{array}{l}\text { MR-BART } \\
\text { [41] } \\
\text { SLDRT [30] } \\
\text { DABE [56] }\end{array}$ & $\checkmark$ & $\checkmark$ & $\checkmark$ & $\checkmark$ & $\begin{array}{l}\text { High } \\
\text { High }\end{array}$ & $\begin{array}{l}\text { Varies } \\
\text { High }\end{array}$ & $\begin{array}{l}\text { It estimates using inter-packet } \\
\text { strain and Kalman filters. } \\
\text { Available bandwidth } \\
\text { measurement was done using } \\
\text { a stable one-way delay. } \\
\text { It uses a total busy period that } \\
\text { includes the frame intervals, } \\
\text { transmission time, and back- } \\
\text { off duration for channels } \\
\text { within the monitoring period } \\
\text { in a distributed manner. }\end{array}$ \\
\hline 2013 & $\begin{array}{l}\text { GNAPP [8] } \\
\\
\text { EABRRL } \\
\text { [51] }\end{array}$ & $\checkmark$ & $\checkmark$ & $\checkmark$ & $\checkmark$ & High & High & $\begin{array}{l}\text { It is used in both the uplink } \\
\text { and downlink and has a novel } \\
\text { two stage filtering algorithm } \\
\text { for improving the precision of } \\
\text { the measurement. } \\
\text { Queuing delay are computed } \\
\text { using two streams of ICMP } \\
\text { timestamp packet. }\end{array}$ \\
\hline
\end{tabular}




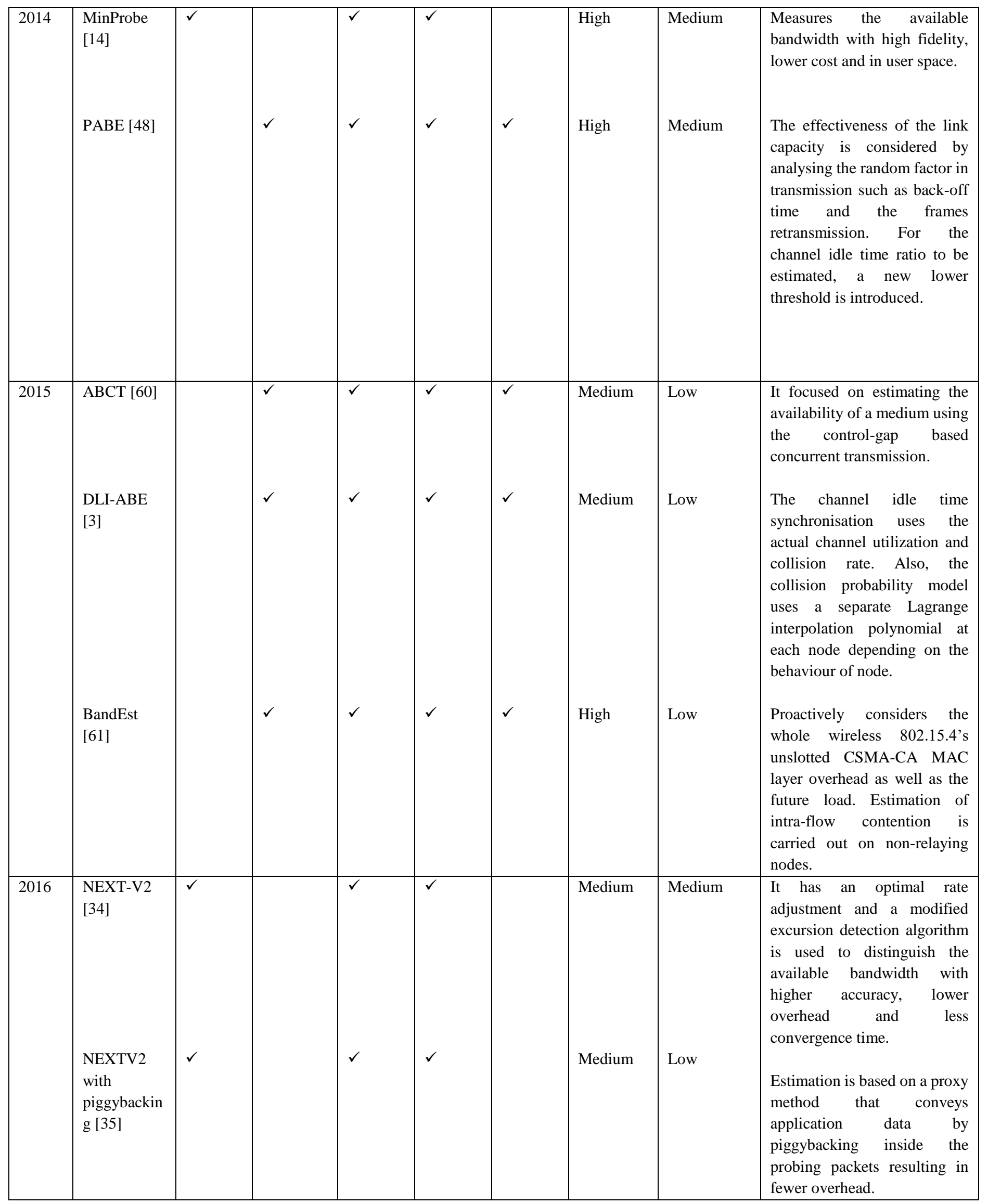




\begin{tabular}{|c|c|c|c|c|c|c|c|}
\hline 2017 & $\begin{array}{l}\text { RT-WABest } \\
\text { [37] }\end{array}$ & $\checkmark$ & $\checkmark$ & $\checkmark$ & Medium & Lo & $\begin{array}{l}\text { It was designed based on } \\
\text { round trip time measurement } \\
\text { with two stage algorithms. The } \\
\text { first stage employs packet pair } \\
\text { dispersion method to measure } \\
\text { the path capacity and the } \\
\text { second stage sends a packet } \\
\text { train to detect the available } \\
\text { bandwidth. }\end{array}$ \\
\hline
\end{tabular}

\section{Proposed Conceptual MANET Bandwidth Estimation for Admission Control Framework}

\subsection{Carrier Sensing Mechanism: Estimating a Nodes Emission Capability}

The Distributed Co-ordination Function (DCF) makes use of carrier sensing multiple access with collision avoidance (CSMA/CA) and the CSMA/CA uses physical carrier sensing and optionally uses the virtual carrier sensing known as the request-to-send/clear-to-send (RTS/CTS) to mitigate the hidden terminals and exposed terminal problems on wireless medium. These problems are common to multi-hop network [62]. The physical carrier sensing is utilized whenever a transmitting node firstly accesses the network. If the bandwidth available on a network is above certain value, the network is termed busy and nodes must wait, otherwise, the channel is assumed to be idle and the node is free to transmit. As previously mentioned, a virtual carrier sensing makes use of a special handshake procedure for channel reservation which is called RTS/CTS mechanism [63]. A carrier sensing range that is unsuitable in a network can affect the interference in MANET, therefore causing a higher collision probability of a channel. When there is a high collision probability on a network, the overhead of that network is affected as well as the whole network of the MANET due to a high demand of network reconnection. Therefore, a challenge is posed to the network designer to implement a network that lower the network overhead by reducing the level of collision probability using a suitable carrier sensing range.

In our conceptual framework, to retrieve the available bandwidth on a carrier sense region, the serviceable bandwidth is estimated. The serviceable bandwidth is defined as the smallest available bandwidth observed on a sensing region. Therefore, Bserv, which is the serviceable bandwidth is estimated as:

$$
B_{\operatorname{serv}(i)}=\underbrace{\min }_{\forall j \in C_{i}}(B(j))
$$

The key idea as regards the bandwidth retrieval process is the use of HELLO message, which is forwarded between nodes for connectivity awareness. The HELLO message only advertises to the first-hop range before it aggregates to the rest of the hops on a network. The serviceable bandwidth calculation remains accurate because the carrier sensing nodes information is aggregated in the packet. This further helps to significantly reduces the overhead within a network, since the HELLO packet is over extended instead of flooding the information throughout the retrieval range. Therefore, information is obtained one after the other during the network deployment process. We present a novel method for estimating the nodes emission capability using the carrier sensing.

\subsection{Measuring the Channel Idle Time Dependency:}

Channel idle time dependency has been proposed in [50], where the author differentiates the BUSY state from the SENSE BUSY states, however, the IDLE state caused by an empty queue is yet to be addressed.

We considered the channel idle time dependency sensed by the sending node and that of the receiving node by differentiating the nodes BUSY state from the SENSE BUSY states and IDLE state caused by an empty queue to ensure accurate available bandwidth estimation for our admission control.

A node is said to be in a state of transmission, only if it is currently emitting signals through its antenna. A node is said to be in a receiving state if there are nodes transmitting within its transmission range. A node is said to be 
in its sensing state if the medium is sensed busy but there is no receiving frame because the energy is below the receiving threshold. A node is said to be in an idle state if it is not transmitting, receiving, or sensing any packet.

We define the BUSY state as a situation whereby a node is in the state of transmission or receiving, while the SENSE BUSY state is defined as a situation whereby a node is in the state of sensing. Any other time apart from the sensing time, the node will be in an IDLE state.

Note that differentiating the SENSE BUSY state from the BUSY state and IDLE state caused by an empty queue has not been researched in the literature. Past works such as [54] have always viewed the SENSE busy state and the BUSY state as the same. While [50] addressed the differentiation of the SENSE BUSY state from the BUSY state, the authors neglected the aspect of regarding the empty queue on a channel as an idle channel time. Therefore, differentiating SENSE busy state from the BUSY state and redefining the idle channel time of a station to include a time that the MAC queue is empty, allows for the synchronization of the sender and the receiver as well as proper available bandwidth estimation.

\section{Conclusion}

This paper reviews the academic literature of available bandwidth estimation methods sand admission control in MANET. Also presented in this work are the subdivision of the bandwidth estimation techniques. Active technique and passive estimation technique are the two main techniques proposed to estimate the available bandwidth for admission control despite different researchers using different names to classify these bandwidth estimation techniques. Classification of bandwidth estimation into active and passive technique helps to simplify the readers understanding of the bandwidth estimation process for admission control. Active end-to-end available bandwidth estimation was thereafter found to introduce extra overhead, affect the accuracy, and degrades the network performance of the bandwidth estimation. Therefore, the active bandwidth estimation approach is not the best choice for admission control in MANET. We have suggested passive available bandwidth estimation for admission control deployment in MANET. Finally, we presented a conceptual framework by proposing a new way of carrier sensing measurement and channel idle time dependency measurement. Future work will include the evaluation and refinement of the framework using real-world data.

\section{References}

1. Hanzo, L and Tafazolli, R., 2009. Admission Control Schemes for 802.11-Based Multi-Hop Mobile Ad hoc Networks: A Survey. IEEE Communications Surveys \& Tutorials, Vol. 11, No. 4, Fourth Quarter

2. L. Hanzo II. and R. Tafazolli, “A Survey of QoS Routing Solutions for Mobile Ad Hoc Networks,” IEEE Commun. Surveys Tutorials, vol. 9, no. 2, pp. 50-70, 2007

3. Chaudhari, S., and Biradar (2015). Available bandwidth estimation using collision probability, idle period synchronization and waiting time. Wireless personal communication an international journal. Vol 83.

4. Lal, C., Laxmi, V., and Gaur, M. (2015). Bandwidth-aware routing and admission control for efficient video streaming over MANETs. Springer Science and business media.

5. Kaur, P and Singh, R., 2013. A Systematic Approach for Congestion Control in Wireless Ad hoc Network Using Opnet.International Journal of Computer Applications, 67 (22).

6. Khoukhi, L., Badis, H., Merghem-Boulahia, L and Esseghir, M., 2013. Admission Control in Wireless ad hoc networks.EURASIP Journal on Wireless Communications and Networking.

7. Arsan, T. (2012). Review of bandwidth estimation tools and application to bandwidth adaptive video streaming. In $9^{\text {th }}$ international conference on high capacity optical networks and enabling technologies (pp. 152-156).

8. Li, M., Wu, Y.-L., \& Chang, C.-R. (2013). Available bandwidth estimation for the network paths with multiple tight links and bursty traffic. Journal of Network and Computer Applications, 36(1), 353-367.

9. Park, H. J., \& Roh, B.-H. (2010). Accurate passive bandwidth estimation (APBE) in IEEE 802.11 wireless LANs. In Proceedings of the 5 th international conference on ubiquitous information technologies and applications (pp. 1-4).

10. Tursunova, S., Inoyatov, K., \& Kim, Y.-T. (2010). Cognitive passive estimation of available bandwidth (cPEAB) in overlapped IEEE 802.11 WiFi WLANs. In IEEE network operations and management symposium (pp. 448-454).

11. Hei, X., Bensaou, B., \& Tsang, D. H. K. (2006). Model-based end-to-end available bandwidth inference using queueing analysis. Computer Networks, 50(12), 1916-1937. 
12. Barzuza, T., Ben Zedeff, S., Modai, O., Vainbrand, L., Wiener, Y., \& Yellin E. (2010). TREND: A dynamic bandwidth estimation and adaptation algorithm for real-time video calling. In 18th international packet video workshop (pp. 126133).

13. Ali, R., \& Zafar, F. (2011). Bandwidth estimation in mobile ad-hoc network (MANET). International Journal of Computer Science, 8(5), 331-337.

14. Wang H., Lee, K., \& Li E. (2014). Timing is Everything: Accurate, Minimum Overhead. Available Bandwidth Estimation in High-speed Wired Networks. Pp. 407-420.

15. Croce, D., \& Leonardi, E. (2011). Large-scale available bandwidth measurements. Interference in current techniques. IEEE Transactions on Network and Service Management, 8(4), 361-374.

16. Xiao, Y., Chen, S., Li, X., \& Li, Y. (2007). A new available bandwidth measurement method based on self-loading periodic streams. In Wireless Communications, Networking and Mobile Computing, WiCom 2007, 21-25 Sept. 2007 (pp. 1904-1907). International Conference on IEEE.

17. Ibrahim, M. F., \& Taib, M. N. (2010). The deployment of end-to-end available bandwidth estimation mechanism in webbased application. In IEEE symposium on industrial electronics and applications (pp. 201-206).

18. Yuan, Z., Venkataraman, H., \& Muntean, G. M. (2009). iBE: A novel bandwidth estimation algorithm for multimedia services over IEEE 802.11 wireless networks. In Proceedings of the 12th IFIP/IEEE international conference on management of multimedia and mobile networks and services: Wired- wireless multimedia networks and services management (Vol. 5842, pp. 69-80).

19. Prasad, R. S., Murray, M., Dovrolis, C., \& Claffy, K. C. (2003). Bandwidth estimation: Metrics. IEEE Network Measurement Techniques, and Tools, 17(6), 27-35.

20. Delphinanto, A., Koonen, T., Zhang, S., \& den Hartog, F. (2010). Path capacity estimation in heterogeneous, best-effort, small-scale IP networks. In IEEE 35th conference on local computer networks (pp. 1076-1083).

21. Pasztor, A., \& Veitch, D. (2002). Active probing using packet quartets. In Proceedings of the $2^{\text {nd }}$ ACM SIGCOMM internet measurement workshop (pp. 293-305).

22. Downey, A. B. (1999). Clink: A tool for estimating internet llink characteristics. http://rocky. wellesley.edu/downey/clink/

23. Downey, A. B. (1999). Using pathchar to estimate internet link characteristics. In ACM SIGCOMM Computer Communication Review (Vol. 29, No. 4, pp. 241-250). ACM

24. Lai, K., \& Baker, M. (2000). Measuring link bandwidths using a deterministic model of packet delay. In Proceedings of the conference on applications, technologies, architectures, and protocols for computer communication (pp. 283-294).

25. Mah, B. A. (2001). Pchar: A tool for measuring internet path characteristics. http://www.employees.org/bmah/Software/pchar/

26. Turrubiartes, M., Torres, D., Angulo, M., \& Munoz, D. (2005). Analysis of IP network path capacity estimation using a variable packet size method. In 15th international conference on electronics, communications and computers (pp. 177182).

27. Guerrero, C. D., \& Labrador, M. A. (2010). Traceband: A fast, low overhead and accurate tool for available bandwidth estimation and monitoring. Computer Networks, 54(6), 977-990.

28. Obara, H., Koseki, S., \& Selin, P. (2012). Packet train pair: A fast and efficient technique for measuring available bandwidth in the internet. In SICE annual conference (pp. 1833-1836).

29. Selin, P., Hasegawa, K., \& Obara, H. (2011). Available bandwidth measurement technique using impulsive packet probing for monitoring end-to-end service quality on the internet. In 17th Asia- Pacific conference on communications (pp. 518-523).

30. Hu, Z., Zhang, D., Zhu, A., Chen, Z., \& Zhou, H. (2012). SLDRT: A measurement technique for available bandwidth on multi-hop path with bursty cross traffic. Computer Networks, 56(14), 3247-3260.

31. Thouin, F., Coates, M., \& Rabbat, M. (2011). Large scale probabilistic available bandwidth estimation. Computer Networks, 55(9), 2065-2078.

32. Li, M., \& Chang, C.-R. (2009). A two-way available bandwidth estimation scheme for multimedia streaming networks adopting scalable video coding. In IEEE Sarnoff symposium (pp. 1-6).

33. Lao, L., Dovrolis, C., \& Sanadidi, M. Y. (2006). The probe gap model can underestimate the available bandwidth of multihop paths. ACM SIGCOMM Computer Communication Review, 36(5), 29-34. 28.

34. Paul, A., Tachibana, A., and Hasegawa, T (2016). An enhanced available bandwidth estimation technique for an end-toend network path. IEEE transaction on network and service management. 13(4). 768-781.

35. Paul, A., Tachibana, A., and Hasegawa, T (2016). Implementation Design of Available Bandwidth Measurement Scheme: A Proxy based Approach. ACM. 257-262.

36. Li, M., Claypool, M., \& Kinicki, R. (2008). WBest: A bandwidth estimation tool for IEEE 802.11 wireless networks. In Proceedings of the 33rd IEEE conference on local computer networks, Montreal, Canada (pp. 374-381). 
37. Yang, T., Jin, Y., Chen. Y., and Jin., Y (2017). RT-WABest: A novel end-to-end bandwidth estimation tool in IEEE 802.11 wireless network. International journal of Distributed sensor networks. 13(2).

38. Hu, N., \& Steenkiste, P. (2002). Estimating available bandwidth using packet pair probing (No. CMUCS- 02-166). CARNEGIE-MELLON UNIV PITTSBURGH PA SCHOOL OF COMPUTER SCIENCE.

39. Hu, N., \& Steenkiste, P. (2003). Evaluation and characterization of available bandwidth probing techniques. IEEE Journal on Selected Areas in Communications, 21(6), 879-894

40. Tunali, T., \& Anar, K. (2006). Adaptive available bandwidth estimation for internet video streaming. Signal Processing: Image Communication, 21(3), 217-234.

41. Sedighizad, M., Seyfe, B., \& Navaie, K. (2012). MR-BART: Multi-rate available bandwidth estimation in real-time. Journal of Network and Computer Applications, 35(2), 731-742.

42. Nam, S. Y., Kim, S., \& Park, W. (2012). Analysis of minimal backlogging-based available bandwidth estimation mechanism. Journal Computer Communications, 35(4), 431-443.

43. Calafate, C. T., Manzoni, P., \& Malumbres, M. P. (2005). Supporting soft real-time services in MANETs using distributed admission control and IEEE 802.11e technology. In 10th IEEE symposium on computers and communications (pp. 217222).

44. Hoang, V. D., Shao, Z., \& Fujise, M. (2006). A new solution to estimate the available bandwidth in MANETs. In IEEE 63rd vehicular technology conference (Vol. 2, pp. 653-657)

45. Ekelin, S., Nilsson, M., Hartikainen, E., Johnsson, A., Mngs, J.-E., Melander, B., et al. (2006). Realtime measurement of end-to-end available bandwidth using Kalman filtering. In Proceedings of the 10th IEEE/IFIP network operations and management symposium.

46. Bergfeldt, E., Ekelin, S., \& Karlsson, J. M. (2009). Real-time available-bandwidth estimation using filtering and change detection. Computer Networks, 53(15), 2617-2645.

47. Lin, H., Liu, M., Zhou, A., Liu, H., \& Li, Z. C. (2010) A novel hybrid probing technique for end-to-end available bandwidth estimation. In IEEE 35th conference on local computer networks (pp. 400-407).

48. Farooq, M., \& Kunz, T. (2013). Proactive Bandwidth Estimation for IEEE 802.15.4-based Networks. in Proceedings of the 77th IEEE Vehicular Technology Conference (VTC '13), pp. 1-5,

49. Gupta Dhruv,WuDi, Mohapatra Prasant, Chuah Chen-Nee. Experimental comparison of bandwidth estimation tools for wireless mesh networks. In :IEEE proceedings ofINFOCOM;October2009.p.2891-95.

50. Zhao, H., Garcia-Palacios, E., Wei, J., \& Xi, Y. (2009). Accurate available bandwidth estimation in IEEE 802.11-based ad hoc networks. Computer Communications, 32(6), 1050-1057.

51. Nam, S. Y., Kim, S. J., Lee, S., \& Kim, H. S. (2013). Estimation of the available bandwidth ratio of a remote link or path segments. Computer Networks, 57(1), 61-77.

52. Brakmo, L. S., \& Peterson, L. L. (1995). TCP Vegas: End-to-end congestion avoidance on a global internet. IEEE Journal on Selected Areas in Communications, 13(8), 1465-1480.

53. Casetti, C., Gerla, M., Mascolo, S., Sanadidi, M. Y., \& Wang, R. (2002). TCP Westwood: Congestion control with faster recovery. Journal of Wireless Networks, 8(5), 467-479.

54. Sarr, C.;Chaudet, C., et al. (2008). Bandwidth Estimation for IEEE 802.11-Based Ad Hoc Networks. IEEE Transactions on Mobile Computing 7(10): 1228-1241.

55. Yan, Z., Dapeng, W., Bin, W., Muqing, W., \& Chunxiu, X. (2008). A novel call admission control routing mechanism for 802.11e based multi-hop MANET. In 4th international conference on wireless communications, networking and mobile computing (pp. 1-4)

56. Peng, Y., \& Yan, Z. (2012). Available bandwidth estimating method in IEEE802.11e based mobile ad hoc network. In $9^{\text {th }}$ international conference on fuzzy systems and knowledge discovery (pp. 2138-2142).

57. de Renesse, R., Ghassemian, M., Friderikos, V., \& Aghvami, A. H. (2004). QoS enabled routing in mobile ad hoc networks. In Fifth IEE international conference on 3G mobile communication technologies (pp. 678-682).

58. Yang, Y., \& Kravets, R. (2005). Contention aware admission control for ad hoc networks. IEEE Transaction on Mobile Computing, 4(4), 363-377.

59. de Renesse, R., Ghassemian, M., Friderikos, V., \& Aghvami, A. H. (2005). Adaptive admission control for ad hoc and sensor networks providing quality of service. Technical Report, King College London.

60. Lei, L., Zhang, T., Zhou, L., Chen, X., Zhang, C., and Luo, C. (2015). Estimating the available medium Access Bandwidth of IEEE 802.11 Ad-hoc Networks with Concurrent Transmission. IEEE Transaction on vehicular technology, 64(2). 689701.

61. Farooq, M., \& Kunz, T. (2015). BandEst: Measurement-based Available Bandwidth Estimation and Flow Admission Control Algorithm for IEEE802.15.4-Based Wireless Multimedia Networks. International Journal of Distributed Sensor Networks. vol 2015. 
62. H. Y. Adarbah, S. Linfoot, B. Arafeh, and A. Duffy, "Effect of Physical and Virtual Carrier Sensing on the AODV Routing Protocol in noisy MANETs," in IEEE International Conference on Consumer Electronics (ICCE), 2013, pp. 508-509

63. N. Vaidya, "On physical carrier sensing in wireless ad hoc networks," in Proceedings IEEE 24th Annual Joint Conference of the IEEE Computer and Communications Societies., 2005, pp. 2525-2535. 